\title{
Results Suggestive of the Brain Trying to 'Re-organize' itself and Recover During the Early Stages of an Epileptic Seizure?
}

\section{Richard Robertson ${ }^{1 *}$}

California State Polytechnic University, Pomona, California, United States

*Corresponding author: Robertson R, Professor Emeritus, California State Polytechnic University, Pomona, California, United States, Tel: 1-909-749-5429; E-mail: cardio38guy@aol.com

Received date: May 16, 2018; Accepted date: June 15, 2018; Published date: June 22, 2018

Copyright: (C2018 Robertson R. This is an open-access article distributed under the terms of the Creative Commons Attribution License, which permits unrestricted use, distribution, and reproduction in any medium, provided the original author and source are credited.

\begin{abstract}
It literally "boggles the mind" to try to imagine the chaos that must be occurring in the brain during an epileptic seizure! No wonder seizures are often referred to as 'brainstorms'! This paper presents some results of my studies analyzing the EEGs of patients during epileptic seizures. Based on cross-frequency coupling strength computations, contour plot snapshots are presented which may be showing the brain 'struggling' to regain control. Results are presented in graphical form for the progression of four epileptic seizures. There are two each from two patients, during the first dozen or two seconds after onset, for seizures lasting roughly a minute. Much longer time studies were done for each in my four recent SSRN e-papers. Using the metric of harmonic wavelet bicoherence, MATLAB contour plots were created illustrating cross-frequency coupling strength among various EEG frequency bands. A short sequence of such plots is presented for each seizure, stepped forward in time by a second (or sometimes less), from a contour plot at or shortly after seizure onset. Each sequence shows how the rather dis-organized and/or sparse pattern of cross-frequency coupling soon after seizure onset morphs into noticeable frequency band coupling. This coupling may be modulated by narrow, low-frequency sub-bands, as suggested by key contour plots. My conjectures may well be premature. But, for these seizures from two different patients with a specific, common type of seizure, complex partial, there seems to be a distinct pattern of activity, with the brain 'fighting' to 'reset' itself, to escape the seizure. There also appears to be a 'characteristic' neuronal spiking frequency for each seizure, which might identify a specific type(s) and location(s) of neurons responsible for this behavior. Perhaps such knowledge could eventually lead to more effective treatment options?
\end{abstract}

Keywords: Cross-frequency coupling; Epileptic seizure; EEG Complex partial seizure; Bicoherence; MATLAB; European Epilepsy Database

\section{Introduction}

This paper presents some results of my studies analyzing the EEGs of patients during epileptic seizures. Based on cross-frequency coupling strength computations, MATLAB contour plot snapshots are presented which may be showing the brain 'struggling' to regain control during the seizure. Results are presented in graphical form for the progression of four epileptic seizures. There are two each from two patients, during the first dozen or two seconds after onset, for seizures lasting roughly three-quarters of to a full minute. Much longer time studies were done for each in my four recent SSRN e-papers [1-4], covering pre-seizure and post-seizure epochs as well.

For these seizures from two different patients with a specific, common type of seizure, complex partial, there seems to be a distinct pattern of activity, with the brain 'fighting' to 'reset' itself, to escape the seizure. There also appears to be a 'characteristic' neuronal spiking frequency for each seizure, possibly for each patient, which might identify a specific type(s) and location(s) of neurons responsible for this. Perhaps such knowledge could eventually lead to more effective treatment options? Much further work needs to be done, though.

Bicoherence, a measure of signal similarity, was used to quantify cross-frequency coupling among different frequency bands of EEG oscillations. To quote from D. Li et al. [5,6], "Bicoherence analysis is an alternative way of quantifying the degree of quadratic phase coupling among different frequency components of a signal, and it is largely independent of the amplitude of the signal." It seemed natural to me to apply their novel, computationally-efficient tool of harmonic wavelet bicoherence to investigate various dynamic aspects of epileptic seizures [7]. I am greatly indebted to Dr. Xiaoli Li and Dr. Duan Li for so generously sharing their MATLAB bicoherence code with me, which I've then modified and extended for my own purposes.

To quote from Buzsaki et al. [8,9], "The cerebral cortex generates multitudes of oscillations at different frequencies, but how the various rhythms interact with each other is not well understood. A wellstudied mechanism is cross-frequency coupling. As described first in the hippocampus, the phase of theta oscillations biases the amplitude of the gamma waves." "While this phase-amplitude (P-A) coupling can reflect spatiotemporal organization of cell assemblies [10], its mechanisms are not well understood. It was hypothesized that P-A coupling between theta and gamma rhythms occurs because perisomatic basket cells contribute to both rhythms by firing theta rhythmic trains of action potentials at gamma frequency [8]. This hypothesis predicts that temporal coordination of both rhythms by the basket cells also introduces a phase-phase (P-P) coupling relationship (or phase synchronization [11]) between theta and gamma oscillations."

"We found reliable phase-phase coupling between theta and both slow gamma and mid-frequency gamma oscillators. We suggest that cross-frequency phase coupling can support multiple time-scale control of neuronal spikes within and across structures." "The precise 
temporal coordination of neuronal spikes at multiple time scales by cross-frequency phase-phase coupling may be beneficial for both transferring information and spike timing-dependent plasticity."

\section{Methods}

For a given 'start time' relative to the identified time of seizure onset and specific electrode location, a 5-sec.-forward segment of EEG data was selected for the bicoherence computations. The result was then treated as the cross-frequency coupling strength at that start time. Stepping ahead by $1 \mathrm{sec}$. (or occasionally less, as was done when contour plots suddenly changed dramatically) thus meant computing bicoherence over a 5-sec. interval with a mid-epoch overlap of $4 \mathrm{sec}$. with the previous one, on a sliding scale.

The contour plots of frequency vs. frequency coupling strength are presented on a 0.5 to $50 \mathrm{~Hz}$ by 0.5 to $50 \mathrm{~Hz}$ matrix, with $1 \mathrm{~Hz}$ step-size for the MATLAB calculations. This range was motivated in part by earlier studies of delta-theta frequency band [5] and theta-slow gamma band [7] cross-frequency couplings. In this paper the theta band is taken to be $4-8 \mathrm{~Hz}$ and slow gamma $30-50 \mathrm{~Hz}$.

Since all four seizures list left temporal lobe scalp electrode location T7 (Figure 1) as one of the sites of seizure origin, all contour plots presented in this study are based on calculations there.

Using actual seizure data from the European Epilepsy Database, I analyzed EEG recordings from two patients in preparing my four recent SSRN e-papers. Papers $[1,3,4]$ concern Patient A, then a 46 yr.old female, who suffered the complex partial seizures investigated. Her consecutive seizures are labeled S10 and S12, corresponding to them occurring within her EED data blocks ( $1 \mathrm{hr}$., at $256 \mathrm{~Hz}$ ) 10 and 12 . Both list her as being awake during the seizures, with 'repetitive spiking' pattern.

Paper [2] concerns Patient B, then a 65 years old male, who suffered the (left temporal lobe) complex partial seizures analyzed. His consecutive seizures are labeled S65 and S69, corresponding to his EED data blocks 65 and 69. Both list him as being awake during the seizures, with 'rhythmic theta wave' pattern for S65 and 'amplitude depression' pattern for S69.

The reader is encouraged to expand the view of individual contour plots to get a more accurate assessment of the influence of specificallymentioned frequencies. The underlying MATLAB figures show even greater detail. The Results and Conjectures are discussed below.

\section{Results}

Figures 2-9 are mostly self-explanatory. The patient studied in each is identified as either Patient A or Patient B, for one of seizures S10 or S12 for A, S65 or S69 for B. Each of these figures is composed of four MATLAB contour plots at various 'start times' relative to that seizure's time of onset; each contour plot is based on EEG data from scalp electrode location T7. These MATLAB graphs illustrate crossfrequency coupling strength, as measured by bicoherence, presented in the form of contour plots over a 0.5 to $50 \mathrm{~Hz}$ by 0.5 to $50 \mathrm{~Hz}$ grid. As the MATLAB colorbar in Figure 2 shows, bicoherence contour regions in dark blue indicate relatively weak cross-frequency coupling, while those tending toward the yellow end of the spectrum demonstrate significantly higher coupling strength. Figures 2-5 are paired together for Patient A, for seizures S10 and S12, respectively. Figures 6-9 are paired together for Patient B, for the respective seizures S65 and S69.

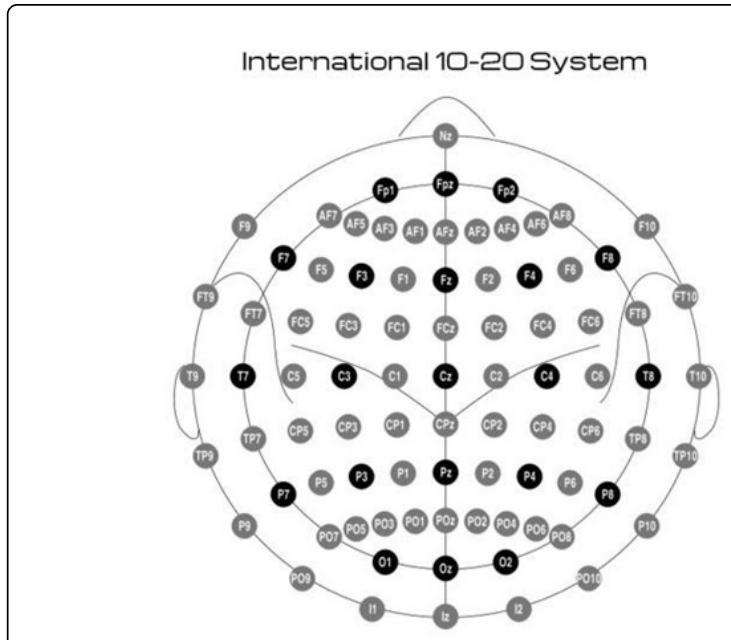

Figure 1: International scalp electrode location labels.

Figure 10 illustrates the contour plots selected as most 'definitive' for each of the four patient-seizure pairings.

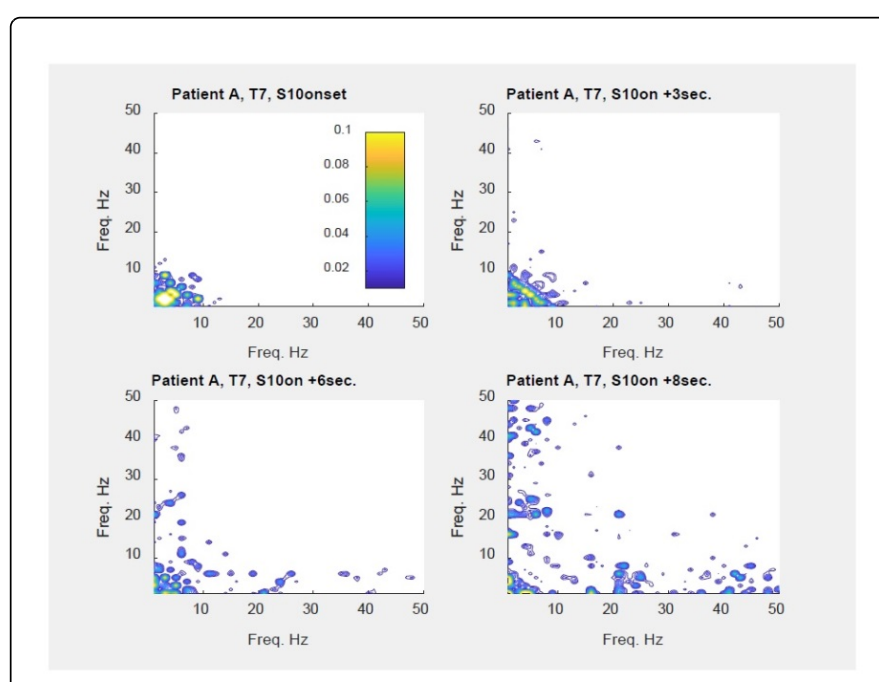

Figure 2: Patient A, for Cross-Frequency Coupling Strength at T7, from Seizure S10 onset to $8 \mathrm{sec}$. afterwards.

The initial intra-seizure contour plot, UL, shows significant coupling only in the $0-10 \mathrm{~Hz}$ frequency corner. A closer, expanded view illustrates strong coupling at $2.5 \mathrm{~Hz}$. The UR plot shows the early stages of wider-spread coupling, with 1,3 , and $5 \mathrm{~Hz}$ influence. In LL, the dominance of $5-6 \mathrm{~Hz}$ has become obvious. LR still shows $5-6 \mathrm{~Hz}$ influence, but with $8 \mathrm{~Hz}$ becoming noticeable, and $21 \mathrm{~Hz}$, too.

UL shows definite coupling strength at 5-6, 8, and 21-22 Hz. UR clearly shows the significance of $8 \mathrm{~Hz}$, with developing $16 \mathrm{~Hz}$ strength. LL paints an even clearer picture of the domination of $8 \mathrm{~Hz}$, with 4 and $16 \mathrm{~Hz}$ strength, too, all of which start fading in LR. 


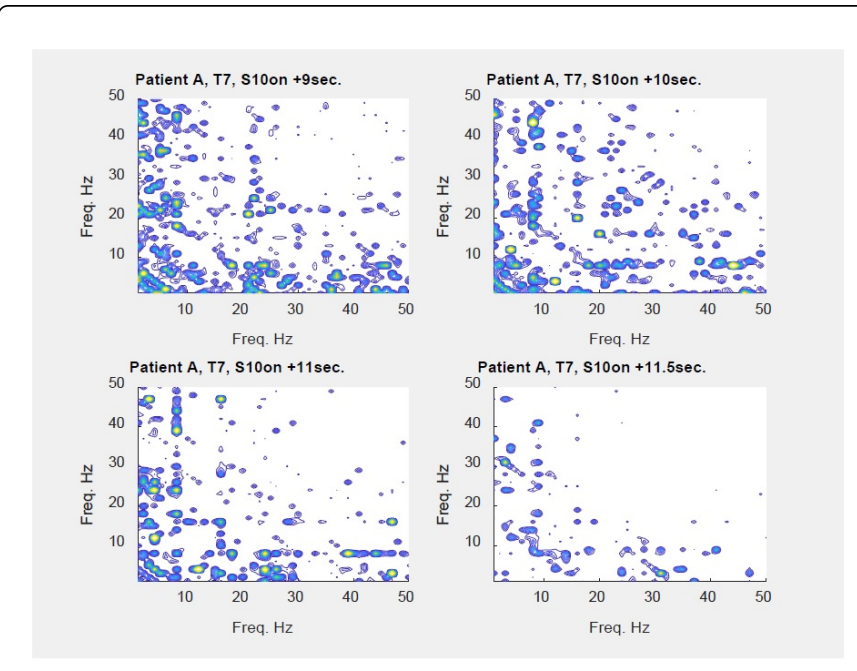

Figure 3: Patient A, for Cross-Frequency Coupling Strength at T7, from 9 to $11.5 \mathrm{sec}$. after $\mathrm{S} 10$ onset.

The initial intra-seizure contour plot, UL, shows noticeable coupling only in the $0-8 \mathrm{~Hz}$ frequency corner, with 1 and $7 \mathrm{~Hz}$ showing some strength. UR shows expanding coupling strength from that corner, especially at 4 and $7 \mathrm{~Hz}$. In LL, 5 and $7 \mathrm{~Hz}$ begin to play more significant roles. 4-5 and $7 \mathrm{~Hz}$ exhibit the strongest coupling in LR.

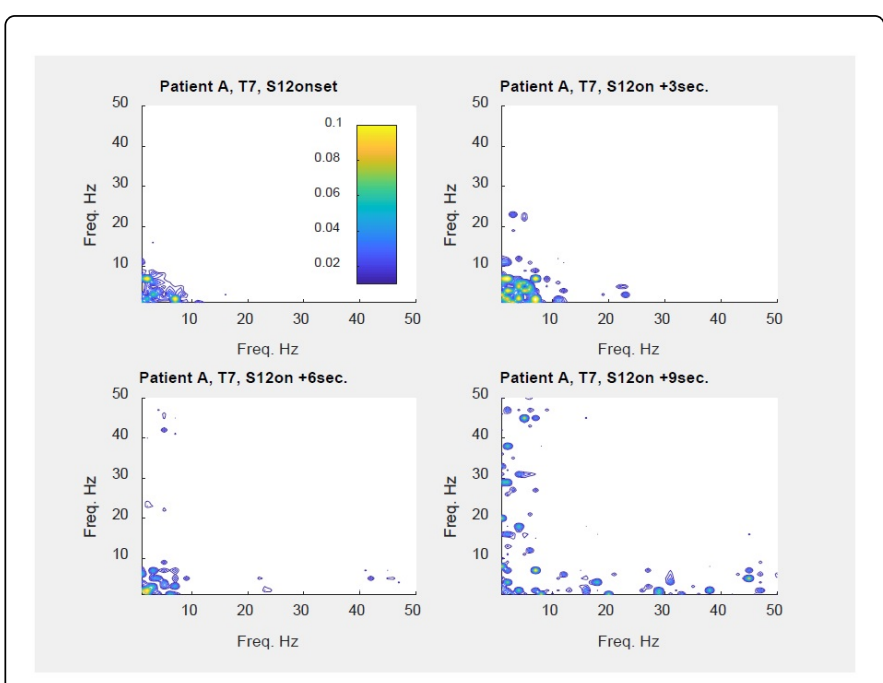

Figure 4: Patient A, for Cross-Frequency Coupling Strength at T7, from Seizure S12 onset to $9 \mathrm{sec}$. afterwards.

Frequencies 1, 4-6, 8, and $16 \mathrm{~Hz}$ show growing coupling strength in UL, even more so in UR. The dominance of $8 \mathrm{~Hz}$ coupling strength is most clear in LL, with some strength at $4-5$ and $16 \mathrm{~Hz}$, too. $8-9 \mathrm{~Hz}$ shows the highest coupling strength in LR, which also displays some 17 $\mathrm{Hz}$ strength.

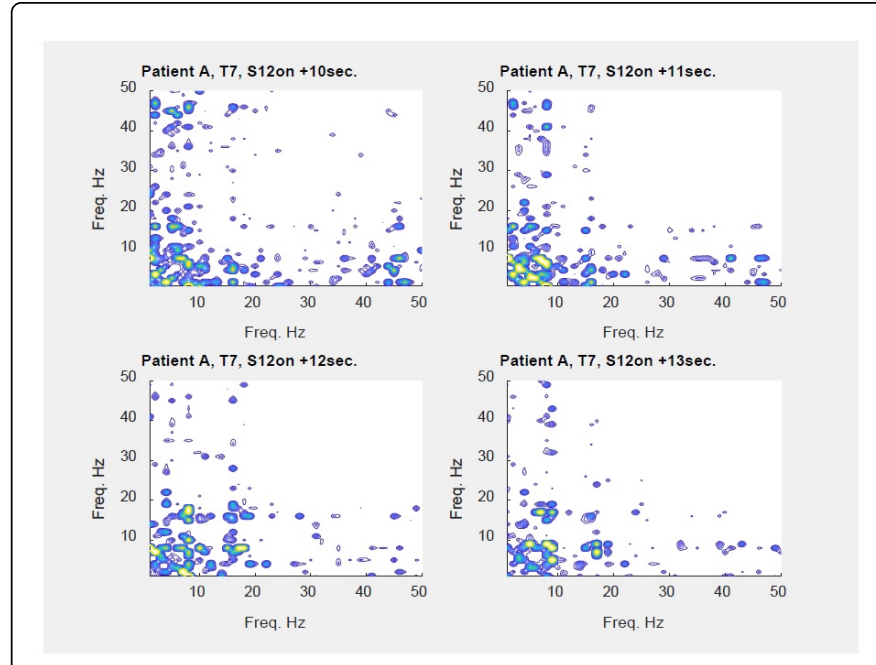

Figure 5: Patient A, for Cross-Frequency Coupling Strength at T7, from 10 to $13 \mathrm{sec}$. after $\mathrm{S} 12$ onset.

What coupling strength is seen in UL occurs at 1-2 and a bit at $7 \mathrm{~Hz}$. A half-sec. later, in UR, the coupling strength at 2 and $5 \mathrm{~Hz}$ becomes obvious. 2, 4 and especially $6 \mathrm{~Hz}$ dominate LL, with a shift back towards 2 and $5 \mathrm{~Hz}$ in LR. Both LL and LR show some coupling at 0.5 and $24 \mathrm{~Hz}$.
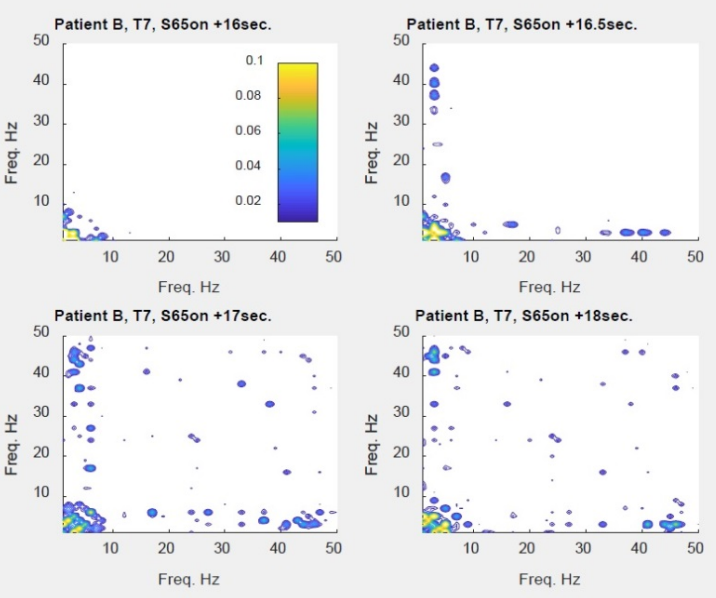

Figure 6: Patient B, for Cross-Frequency Coupling Strength at T7, from 16 to $18 \mathrm{sec}$. after $\mathrm{S} 65$ onset.

$0.5,2.5,5$, and a weak $24 \mathrm{~Hz}$ coupling strength are still apparent in UL, with all showing greater strength in UR and LL, especially at 2.5 $\mathrm{Hz}$. A half-sec. later, in LR, coupling strength appears greatest at 1 and 4-6 Hz. 


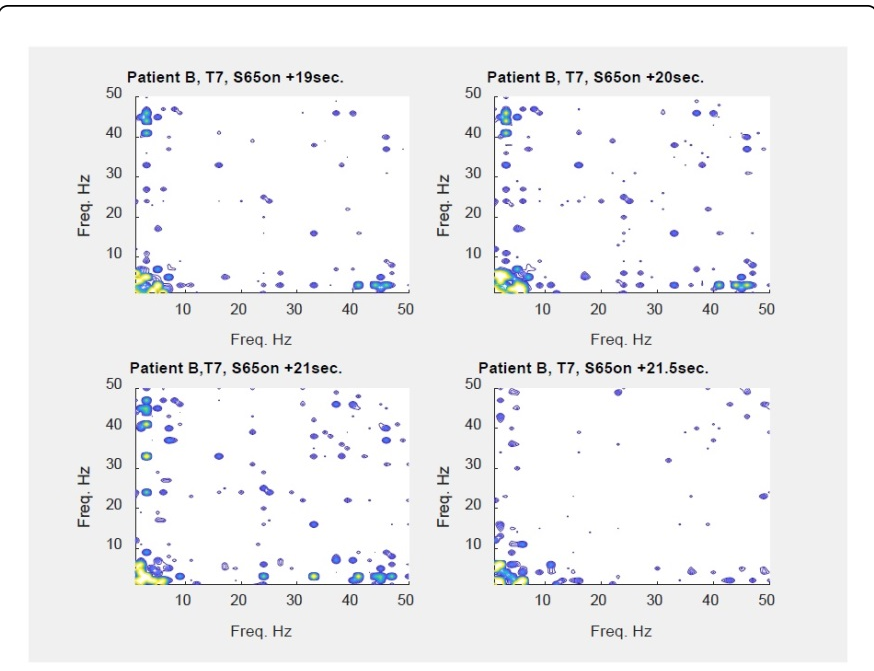

Figure 7: Patient B, for Cross-Frequency Coupling Strength at T7, from 19 to $21.5 \mathrm{sec}$. after $\mathrm{S} 65$ onset.

Almost no organization of frequency couplings is evident at onset, in UL, except a bit at 1 and $32 \mathrm{~Hz} .6 \mathrm{sec}$. later, in UR, some is seen at 0.5 and $40 \mathrm{~Hz}$. In LL, coupling strength is observed at 0.5 and $5-7 \mathrm{~Hz}$. Coupling strength 2 sec. later, in LR, is seen at $0.5,2,5-6$ and $37 \mathrm{~Hz}$.

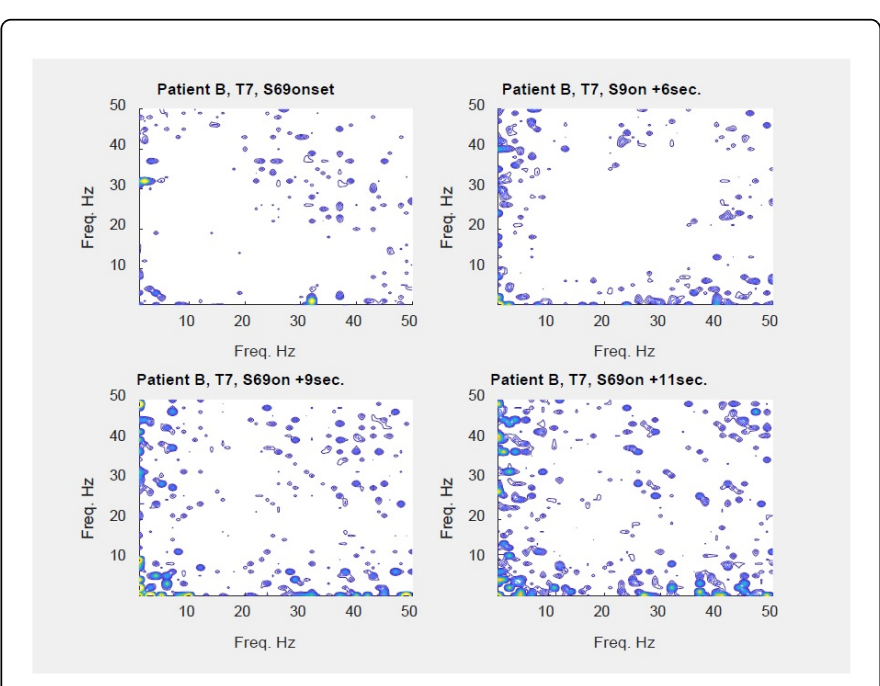

Figure 8: Patient B, for Cross-Frequency Coupling Strength at T7, from Seizure 569 onset to $11 \mathrm{sec}$. afterwards.

Coupling strength in UL is seen at 0.5 and $2.5 \mathrm{~Hz}$, weakly so at 1,27 , and $40 \mathrm{~Hz}$, but is greatest at $5 \mathrm{~Hz}$. UR demonstrates coupling at 1,2.5, and $27-28 \mathrm{~Hz}$, but, again, the strongest is seen at $5 \mathrm{~Hz}$. LL shows a somewhat stronger version of those frequency couplings than in UR, along with some strength at 8 and $10 \mathrm{~Hz}$. LR also shows coupling strength at $2.5,5,8,10$, and $27-28 \mathrm{~Hz}$, though mostly weaker than in LL.

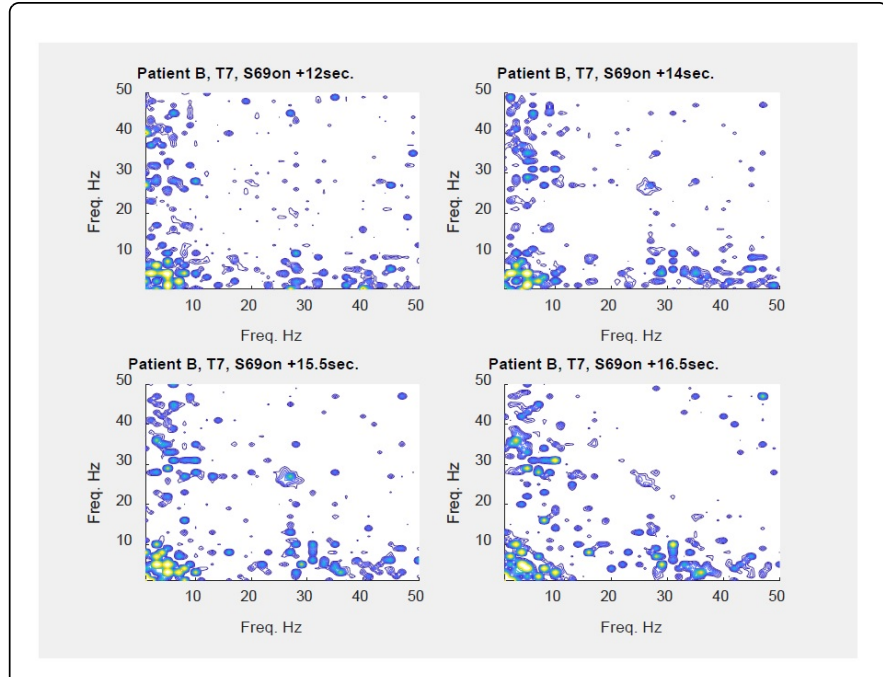

Figure 9: Patient B, for Cross-Frequency Coupling Strength at T7, from 12 to $16.5 \mathrm{sec}$. after $\mathrm{S} 69$ onset.

Each of the four paired Figures 2-9 presents a sequence of eight contour plots of cross-frequency coupling strength. Each of these pairs, covering one of two seizures each for both patients, follows an individual sequence of eight consecutive start times, in small timesteps from seizure onset, or just after, to a fraction of the way toward seizure offset.

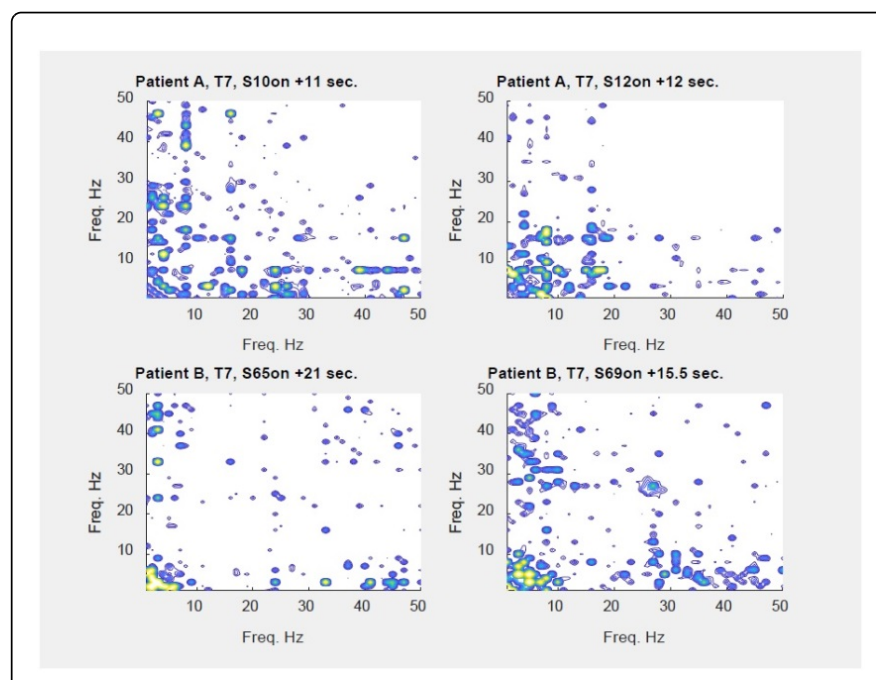

Figure 10: 'Characteristic' Frequency Coupling-Strength patterns for all four patient-seizure pairings.

The sequence of eight contour plots for each of the four patientseizure pairings suggests a progression from an initial rather disorganized and/or sparse demonstration of cross-frequency coupling strength. This is true up through the $7^{\text {th }}$ contour plot, selected as the most definitive for each pairing. Figure 10 presents these four key contour plots, each representing a separate patient-seizure pairing with its own time scale relative to its seizure's onset. Incidentally, the chosen $8^{\text {th }}$ plot for each show an overall coupling strength weaker than its predecessor. 
Citation: Robertson R (2018) Results Suggestive of the Brain Trying to 'Re-organize' itself and Recover During the Early Stages of an Epileptic Seizure?. Epilepsy J 4: 1000127. doi:10.4172/2472-0895.1000127

Page 5 of 5

\section{Conjectures}

In Figure 10's Upper Left corner, for the Patient A-seizure S10 pair, the coupling-strength domination of the $8 \mathrm{~Hz}$ frequency is apparent, with strength also seen at 4 and $16 \mathrm{~Hz}$. From my perspective, the unusually 'rigid' horizontal/vertical patterns seen at 8 and $16 \mathrm{~Hz}$ seem to have emerged from the seizure's initial relative chaos, and perhaps demonstrate the brain's attempt to 're-organize' or 'reset' itself, at least near T7.

In Figure 10's Upper Right corner, for the Patient A-S12 pair, the same basic comments can be made regarding 8,4 , and $16 \mathrm{~Hz}$, though the patterns are a bit more 'shadowy', less 'rigid', somewhat weaker than in A-S10. Nevertheless, $8 \mathrm{~Hz}$ seems to be a 'characteristic', perhaps modulating or even 'resetting', frequency for Patient A's two seizures.

In Figure 10's Lower Left corner, for the Patient B-seizure S65 pair, the coupling-strength domination of the $2.5 \mathrm{~Hz}$ frequency is apparent, with strength also seen at 5 and $24 \mathrm{~Hz}$.

In Figure 10's Lower Right corner, for the Patient B-S69 pair, the dominant coupling-strength frequency is $2.5 \mathrm{~Hz}$, along with coupling strength at its harmonics of 5, 10, and $27.5 \mathrm{~Hz}$. So, for Patient B's two seizures, $2.5 \mathrm{~Hz}$ seems to be a 'characteristic' frequency.

\section{References}

1. Robertson R (2017) Measuring Cross-Frequency Coupling of EEG Signals between Different Scalp Electrode Locations during Extended Seizure Epochs.
2. Robertson R (2017) A Novel Method for Illustrating Seizure Dynamics During Eight Hours of a Patient Experiencing Multiple Ictal Events.

3. Robertson R (2017) Cross-Frequency Phase: Phase Coupling of EEG Signals During Near-Seizure Epochs.

4. Robertson R (2018) Using Contour Plots of Bicoherence to Illustrate and Investigate Cross-Frequency Coupling of EEG Oscillations.

5. Li D, Li X, Hagihira S, Sleigh JW (2013) Cross-frequency coupling during isoflurane anaesthesia as revealed by electroencephalographic harmonic wavelet bicoherence. British J Anaesthe 110: 409-419.

6. Li X, Li D, Voss LJ, Sleigh JW (2009) The comodulation measure of neuronal oscillations with general harmonic wavelet bicoherence and application to sleep analysis. NeuroImage 48: 501-514.

7. Belluscio MA, Mizuseki K, Schmidt R, Kempter R, Buzsáki G (2012) Cross-Frequency Phase-Phase Coupling between Theta and Gamma Oscillations in the Hippocampus. J Neurosci 32: 423-435.

8. Buzsáki G, Leung LW, Vanderwolf CH (1983) Cellular bases of hippocampal EEG in the behaving rat. Brain Res 287: 139-171.

9. Buzsáki G, Buhl DL, Harris KD, Csicsvari J, Czéh B, et al. (2003) Hippocampal network patterns of activity in the mouse. Neuroscience 116: 201-211.

10. Buzsáki G (2010) Neural syntax: cell assemblies, synapsembles, and readers. Neuron 68: 362-385.

11. Tass P, Rosenblum MG, Weule J, Kurths J, Pikovsky A, et al. (1998) Detection of $\mathrm{n}: \mathrm{m}$ phase locking from noisy data: application to magnetoencephalography. Phys Rev Lett 81: 3291-3294. 